Provided for non-commercial research and education use. Not for reproduction, distribution or commercial use.

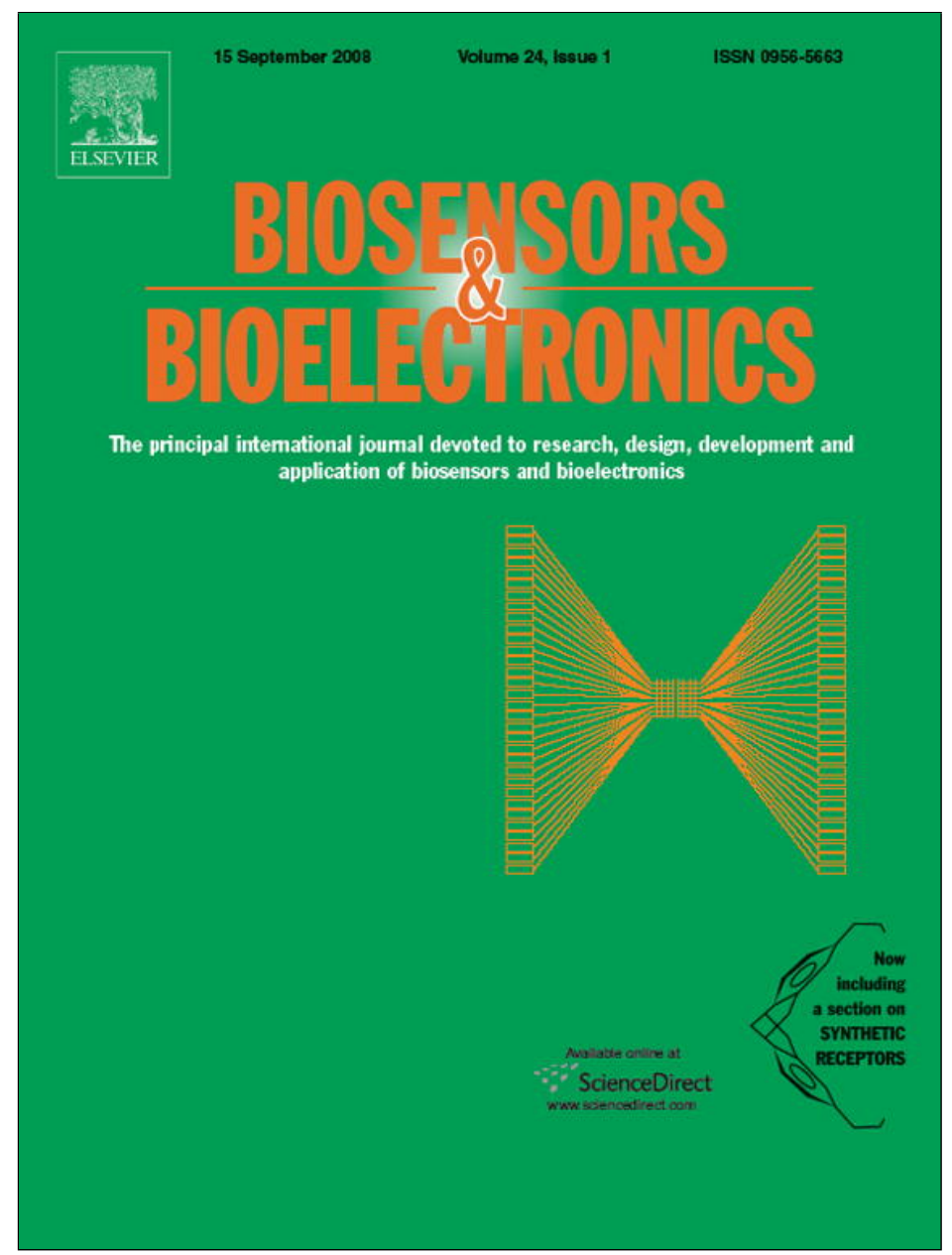

This article appeared in a journal published by Elsevier. The attached copy is furnished to the author for internal non-commercial research and education use, including for instruction at the authors institution and sharing with colleagues.

Other uses, including reproduction and distribution, or selling or licensing copies, or posting to personal, institutional or third party websites are prohibited.

In most cases authors are permitted to post their version of the article (e.g. in Word or Tex form) to their personal website or institutional repository. Authors requiring further information regarding Elsevier's archiving and manuscript policies are encouraged to visit:

http://www.elsevier.com/copyright 


\title{
The application of neoglycopeptides in the development of sensitive surface plasmon resonance-based biosensors
}

\author{
C. Elizabeth P. Maljaars ${ }^{\mathrm{a}}$, Adriana Carvalho de Souza ${ }^{\mathrm{a}}$, Koen M. Halkes ${ }^{\mathrm{a}}$, Peter J. Upton ${ }^{\mathrm{b}}$, \\ Stuart M. Reeman ${ }^{\mathrm{b}}$, Sabine Andréc ${ }^{\mathrm{c}}$, Hans-Joachim Gabius ${ }^{\mathrm{c}}$, \\ Martin B. McDonnell ${ }^{\mathrm{b}}$, Johannis P. Kamerling ${ }^{\mathrm{a}, *}$ \\ a Bijvoet Center, Department of Bio-Organic Chemistry, Utrecht University, Padualaan 8, 3584 CH Utrecht, The Netherlands \\ ${ }^{\mathrm{b}}$ Dstl, Porton Down, Salisbury, Wiltshire SP4 0JQ UK \\ ${ }^{\mathrm{c}}$ Institute of Physiological Chemistry, Faculty of Veterinary Medicine, Ludwig-Maximilians-University Munich, Veterinärstr. 13, 80539 Munich, Germany
}

\section{A R T I C L E I N F O}

\section{Article history:}

Received 3 December 2007

Received in revised form 25 February 2008

Accepted 17 March 2008

Available online 22 March 2008

\section{Keywords:}

Agglutinin

Alkanethiol self-assembled monolayers

Carbohydrate-binding proteins

Galectin-1

Glycopeptides

Surface plasmon resonance

\begin{abstract}
A B S T R A C T
The development of a biosensor based on surface plasmon resonance is described for the detection of carbohydrate-binding proteins in solution on a Biacore 2000 instrument, using immobilized glycopeptides as ligands. Their selection was based on previous screenings of solid-phase glycopeptide libraries with Ricinus communis agglutinin $\left(\mathrm{RCA}_{120}\right)$ and human adhesion/growth-regulatory galectin-1 (h-Gal-1). Glycopeptides were immobilized on Au sensor chips functionalized with mixed self-assembled monolayers of different ratios of 11-mercapto-1-undecanol and 11-mercaptoundecanoic acid, and of 3-mercapto-1propanol and 11-mercaptoundecanoic acid. The biosensors were optimized for the detection of RCA , $_{120}$, and a detection limit of $0.13 \mathrm{nM}$ was obtained. Subsequent experiments with h-Gal-1 indicated a detection limit of at least $0.9 \mathrm{nM}$ for this lectin. Additionally, the effect of interfering proteins on the sensitivity of the optimized biosensor was investigated.
\end{abstract}

(c) 2008 Elsevier B.V. All rights reserved.

\section{Introduction}

Since the development of the first biosensors in the early 1960s (Clark Jr. and Lions, 1962) this research area has seen an enormous growth (Cullum and Vo-Dinh, 2000; Lu and Rosenzweig, 2000; VoDinh and Cullum, 2000). Nowadays, biosensors are widely applied for the detection, identification, and characterization of biological material, which is of great importance in industrial (Castillo et al., 2004), medical (Hsieh et al., 2004; Dillon et al., 2005; Jiang et al., 2005), food (Gustavsson et al., 2002; Haasnoot et al., 2002; Indyk and Filonzi, 2005; Muller-Renaud et al., 2005), and environmental (Castillo et al., 2004) analysis. Surface plasmon resonance-based biosensors are currently widely used to monitor these interactions (Chou et al., 2004; Oh et al., 2004; Choi et al., 2005). Although analytes can be detected by a wide range of immobilized ligands, such as antibodies (Soh et al., 2003; Liu et al., 2004; Choi et al., 2005; Muller-Renaud et al., 2005), enzymes, and carbohydrate-binding proteins (CBPs) (Hsieh et al., 2004), the development of carbohydrate-based biosensors has

\footnotetext{
* Corresponding author. Tel.: +31 302533479 ; fax: +31 302540980 . E-mail address: j.p.kamerling@uu.nl (J.P. Kamerling).
}

recently received increased attention because of the importance of carbohydrate-protein interactions in life. So far, carbohydratebased biosensors are predominantly used for the characterization of the kinetics and affinity of carbohydrate-protein interactions (Shinohara et al., 1994; Bourne et al., 2002; Zhang et al., 2006), or in an array-type set-up (Love and Seeberger, 2002; Feizi et al., 2003; Blixt et al., 2004; Dyukova et al., 2006) to determine the specificity, and possible cross-reactivity of a CBP. However, it would also be of interest to apply carbohydrate-based biosensors to the sensitive detection and quantification of minute amounts of CBPs, which could lead to the development of diagnostic tools.

The access to structurally well-defined carbohydrates is complicated, since both the isolation from natural sources, and the synthesis are very challenging. Since it has been demonstrated that complex carbohydrates can be efficiently mimicked by glycopeptides (St. Hilaire et al., 1998; Halkes et al., 2003; Maljaars et al., 2006), here, the development of a SPR-based biosensor using immobilized glycopeptides is described for the detection of CBPs. The glycopeptides are lead structures that were obtained from the systematic screening of solid-phase glycopeptide libraries with Ricinus communis agglutinin ( $\mathrm{RCA}_{120}$; Maljaars et al., 2006) and human adhesion/growth-regulatory galectin-1 (h-Gal-1; André et al., 2007a). In the present study, these glycopeptides are used for the 
set-up of a biosensor system for the sensitive detection of $\mathrm{RCA}_{120}$ and h-Gal-1 in solution on a Biacore 2000 instrument. In addition, the performance of the optimized biosensors was investigated in the detection of low amounts of $\mathrm{RCA}_{120}$ and h-Gal-1 in complex protein mixtures.

\section{Materials and methods}

\subsection{General}

Bovine serum albumin (BSA), Ricinus communis agglutinin $\left(\mathrm{RCA}_{120}\right)$, bovine ribonuclease $\mathrm{B}$ (RNase $\mathrm{B}$ ), ovalbumin, trypsin inhibitor, 11-mercaptoundecanoic acid (11-MUA), 11-mercapto1-undecanol (11-MUOH), 3-mercapto-1-propanol (3-MPOH), $\mathrm{N}$-ethyl- $\mathrm{N}^{\prime}$-(dimethylaminopropyl)carbodiimide hydrochloride (EDC), and $N$-hydroxysuccinimide (NHS) were obtained from Sigma-Aldrich (Zwijndrecht, The Netherlands); human Galectin1 (h-Gal-1) was prepared, purified, and quality controlled as described previously (André et al., 2004). The SIA-kit AU was purchased from Biacore AB (Uppsala, Sweden). All solvents were of HPLC grade and were used without further purification.

\subsection{Preparation of $\mathbf{N}$-acetylated glycopeptides $\mathbf{1}$ and $\mathbf{2}$}

Fmoc-protected (9-fluorenylmethoxycarbonyl) glycopeptides A(Gal-)CYPY(Gal-)C and (Lac-)TMRA(Lac-)TCha were previously prepared on Wang-resin prefunctionalized with a Rink-amide linker and an Ionization Mass Peptide (IMP)spacer (GPPFPFK) (Maljaars et al., 2006; André et al., 2007a). The resin-bound glycopeptides were treated with $20 \%$ piperidine in $N, N$-dimethylformamide $(2 \times, 2+18 \mathrm{~min})$ to remove the Fmoc-protecting group, and the resin was washed with $\mathrm{N}, \mathrm{N}$ dimethylformamide $(10 \times)$. Subsequently, the N-termini were acetylated using $20 \%$ acetic anhydride in $\mathrm{N}, \mathrm{N}$-dimethylformamide ( $15 \mathrm{~min})$. The resin was washed with $\mathrm{N}, \mathrm{N}$-dimethylformamide $(6 \times)$ and methanol $(4 \times)$, and the glycopeptides were de-Oacetylated overnight with hydrazine monohydrate $(55 \mu \mathrm{l})$ in methanol $(1 \mathrm{ml})$, followed by washings with methanol $(6 \times)$ and water $(4 \times)$. The amino acid side-chains were deprotected with concomitant release of the glycopeptides from the resin by using a mixture of trifluoroacetic acid/water/triisopropylsilane (95:2.5:2.5; $4 \times 30 \mathrm{~min})$. The cleaved glycopeptides $\mathbf{1}$ and $\mathbf{2}$ were extracted from the resin with $10 \%$ aq. acetonitrile $(4 \times)$, then concentrated, and purified by reverse-phase HPLC. Then, the glycopeptides were lyophilized, and analyzed by MALDI-TOF MS (1, AcA(Gal-)CYPY(Gal-)C-GPPFPFK, $[\mathrm{M}+\mathrm{H}]_{\text {calcd }}=1854.797 \mathrm{Da}$, $[\mathrm{M}+\mathrm{H}]_{\mathrm{det}}=1854.841 \mathrm{Da} ; \quad$ 2, Ac(Lac-)TMRA(Lac-)TCha-GPPFPFK, $[\mathrm{M}+\mathrm{H}]_{\text {calcd }}=2192.057,[\mathrm{M}+\mathrm{H}]_{\mathrm{det}}=2192.424$.

\subsection{Surface plasmon resonance}

\subsubsection{Surface preparation}

Self-assembled monolayers (SAMs) of alkanethiols were created on Au-surfaces from a SIA-kit Au, in order to prepare SAMs derivatized with glycopeptides 1 and $\mathbf{2}$ (Choi et al., 2005; Lee et al., 2005). To this end, $9 \mathrm{mM}$ mixtures of $11-\mathrm{MUOH} / 11-\mathrm{MUA}$ (molar ratios: 95:5, 9:1, and 8:2), and 3-MPOH/11-MUA (molar ratio: 9:1) were prepared in absolute ethanol. The Au-surfaces were immersed in the appropriate alkanethiol mixture for $24 \mathrm{~h}$. Then, the Au-surfaces were washed with absolute ethanol and deionized water, and dried with a stream of $\mathrm{N}_{2}$ prior to the assembly of the sensor chips.

All surface plasmon resonance (SPR)-measurements were performed on a Biacore 2000 instrument. All reagents for the surface preparations were injected at a flow rate of $5 \mu \mathrm{min}^{-1}$, using HBSEP (10 mM 2-[4-(2-hydroxyethyl)-1-piperazinyl]-ethanesulfonic acid (HEPES) buffer, pH 7.5, containing $150 \mathrm{mM} \mathrm{NaCl}, 0.005 \%$ polyoxyethylenesorbitan (p20), and $3 \mathrm{mM}$ ethylenediaminetetraacetic acid (EDTA)) as the running buffer. The carboxyl-terminated alkanethiols on the surfaces of the appropriate flow cell of each sensor chip were activated by the injection of $35 \mu$ l of a $1: 1$ mixture of $0.05 \mathrm{M}$ NHS and $0.2 \mathrm{M} \mathrm{EDC}$, and the appropriate glycopeptide $\left(3 \times 35 \mu \mathrm{l}, 500 \mu \mathrm{g} \mathrm{ml}^{-1}\right.$ in $10 \mathrm{mM} \mathrm{NaHCO} 3$ buffer, $\mathrm{pH}$ 8.5) was injected. The remaining $N$-hydroxysuccinimide esters were blocked by injection of $1.0 \mathrm{M}$ ethanolamine hydrochloride, pH $8.5(35 \mu \mathrm{l})$. Using this protocol, six SAMs were prepared: SAM-1: 8:2 11-MUOH/11-MUA, 700 RU glycopeptide 1; SAM2: 9:1 11-MUOH/11-MUA, 510 RU glycopeptide 1; SAM-3: 95:5 11-MUOH/11-MUA, 300 RU glycopeptide 1; SAM-4: 9:1 3-MPOH/11-MUA, 930 RU glycopeptide 1; SAM-5: 9:1 3-MPOH/11MUA, 1256 RU glycopeptide 2; SAM-6: 9:1 3-MPOH/11-MUA, 1032 RU glycopeptide $\mathbf{1 .}$

\subsubsection{Detection measurements}

The interaction experiments with soluble $\mathrm{RCA}_{120}$ and h-Gal-1, and the immobilized glycopeptides were carried out in duplicate using a $10 \mathrm{mM}$ HBS-EP running buffer, $\mathrm{pH}$ 7.5. $\mathrm{RCA}_{120}$ and h-Gal1 were injected into the appropriate flow cells for $3 \mathrm{~min}$, allowing $3 \mathrm{~min}$ for dissociation, and using a flow rate of $5 \mu \mathrm{min}^{-1}$.

For the experiments with glycopeptide 1 on SAM-1, -2, and $\mathbf{- 3}$, the $\mathrm{RCA}_{120}$-concentration range covered was $41.7-0.08 \mathrm{nM}$, obtained by 2 -fold dilution. For SAM-4 and SAM-6, an $\mathrm{RCA}_{120^{-}}$ concentration range of 4.2-0.008 $\mathrm{nM}$ was used. For the experiments with immobilized glycopeptide 2 (SAM-5), the h-Gal-1concentration range covered was $500-0.9 \mathrm{nM}$. The surfaces were regenerated with a 2 -min pulse of a solution containing a mixture of $200 \mathrm{mM}$ lactose and $0.002 \%$ sodium dodecyl sulfate. All data were double-reference-subtracted. The detection limit of the assay was defined as the lowest concentration that could be visually discerned from the overlapping lower concentrations. Calibration curves of the duplicate experiments on SAM-1-4 were created by plotting $\mathrm{dRU} / \mathrm{dt}$ versus the $\mathrm{RCA}_{120}$ concentration; $\mathrm{dRU} / \mathrm{dt}$ was calculated for the initial $30 \mathrm{~s}$ of the association phase, using $R U_{\text {average }}$ of 3 datapoints around $t=30 \mathrm{~s}$ and around $t=0$. The average of the duplicates of each datapoint was used to fit a trendline. For h-Gal-1, $K_{\mathrm{D}}$ could be determined from non-linear fitting of the plot of the response at equilibrium $\left(R_{\text {eq }}\right)$ versus the h-Gal-1 concentration using the average model with steady state affinity in BIAevaluation 4.1.

\subsubsection{Detection of analyte in a complex protein mixture}

Two stock solutions of a protein mixture were prepared in HBSEP buffer, pH 7.5, by mixing equal volumes of 10 or $100 \mu \mathrm{M}$ BSA, ovalbumin, RNase $B$, and trypsin inhibitor. These stock solutions were used in the appropriate amounts to prepare a $4.2 \mathrm{nM} \mathrm{RCA}_{120}$ solution containing a 10-, 100-, 1000-, or 10,000-fold molar excess of each component of the protein mixture. Subsequently, dilution series (4.2-0.008 nM) of $\mathrm{RCA}_{120}$ were prepared by 2-fold dilution. The above-described stock solutions of the protein mixture were

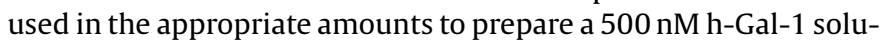
tion containing an equimolar, or a $10-, 50-$, or 100 -fold molar excess of each component of the protein mixture. The dilution series of h-Gal-1 (500-0.9 nM) was prepared as described for $\mathrm{RCA}_{120}$. The surfaces were regenerated with a 2-min pulse of a solution containing a mixture of $200 \mathrm{mM}$ lactose and $0.002 \%$ sodium dodecyl sulfate, and all data were double-reference-subtracted.

\section{Results and discussion}

\subsection{Modification of the glycopeptides}

The non- $\mathrm{N}$-acetylated analogs of the glycopeptides used in this study were identified in previous screenings of solid-phase gly- 
Table 1

The different glycopeptide-derivatized SAMs and the observed detection limits for $\mathrm{RCA}_{120}$ and h-Gal-1

\begin{tabular}{llllc}
\hline SAM & Composition & Ligand & Analyte & Immobilization level (RU) \\
\hline SAM-1 & $8: 2$ 11-MUOH/11-MUA & $\mathbf{1}$ & RCA $_{120}$ & 700 \\
SAM-2 & $9: 111-\mathrm{MUOH} / 11-\mathrm{MUA}$ & $\mathbf{1}$ & RCA $_{120}$ & 510 \\
SAM-3 & $95: 511-\mathrm{MUOH} / 11-\mathrm{MUA}$ & $\mathbf{1}$ & RCA $_{120}$ & 300 \\
SAM-4 & $9: 13-\mathrm{MPOH} / 11-\mathrm{MUA}$ & $\mathbf{1}$ & $\mathrm{RCA}_{120}$ & 930 \\
SAM-5 & $9: 13-\mathrm{MPOH} / 11-\mathrm{MUA}$ & $\mathbf{2}$ & $\mathrm{h}-\mathrm{Gal}-1$ & 1256 \\
SAM-6 & $9: 13-\mathrm{MPOH} / 11-\mathrm{MUA}$ & $\mathbf{1}$ & RCA $_{120}$ & 1032 \\
\hline
\end{tabular}

Ligand 1: AcA(Gal-)CYPY(Gal-)C-GPPFPFK. Ligand 2: Ac(Lac-)TMRA(Lac-)TCha-GPPFPFK.

copeptide libraries using fluorescently labeled $\mathrm{RCA}_{120}$ and h-Gal-1 (Maljaars et al., 2006; André et al., 2007a). For the present experiments, $N$-acetylated forms were used (1, AcA(Gal-)CYPY(Gal-)CGPPFPFK; 2, Ac(Lac-)TMRA(Lac-)TCha-GPPFPFK). This allowed a homogeneous orientation of the glycopeptides, by using the amine function in the side-chain of Lys, which is the C-terminal residue in the IMP-spacer, for the coupling of the glycopeptides to the sensor chips.

\subsection{Detection of $R C A_{120}$ on $\mathbf{S A M - 1 , - 2 , - 3}$, and $\mathbf{- 4}$}

Previously, it has been shown that sensitive biosensors can be prepared using self-assembled monolayers of alkanethiols on a gold surface, to which a ligand can be coupled (Miura et al., 1999; Ista et al., 2001; Tamada et al., 2001; Choi et al., 2005; Lee et al., 2005). It was demonstrated that SAMs containing a mixture of hydroxyl- and carboxyl-terminated alkanethiols provide a better sensitivity than SAMs containing acid thiols only. Therefore, SAMs were created by immersing Au-surfaces in alkanethiol solutions containing different molar ratios of 11-mercapto-1-undecanol (11-MUOH) and 11-mercaptoundecanoic acid (11-MUA), or 3mercapto-1-propanol (3-MPOH) and 11-MUA. Glycopeptide 1 was

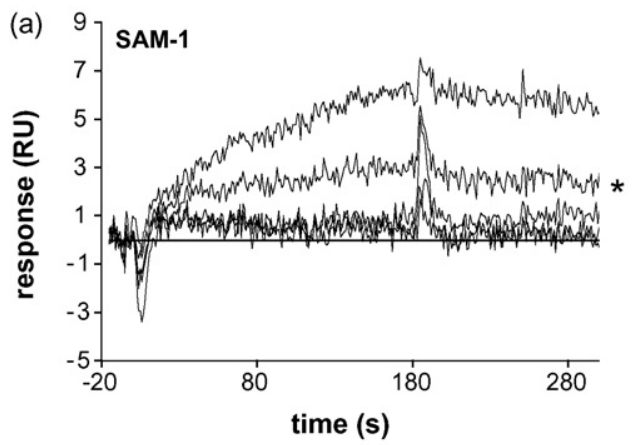

immobilized on Au-chips decorated with 11-MUOH and 11-MUA in the molar ratios 8:2 (SAM-1), 9:1 (SAM-2), and 95:5 (SAM-3) (Table 1).

Binding data were collected at different concentrations (41.7-0.08 nM) at a flow rate of $5 \mu \mathrm{min}^{-1}$. Since initial experiments did not indicate any non-specific binding of $\mathrm{RCA}_{120}$ to a reference surface with underivatized 11-MUOH and 11-MUA on the respective SAM, this surface was used as the reference surface. Examination of the concentration-dependent overlay plots of $\mathrm{RCA}_{120}$ binding to glycopeptide 1-derivatized SAM-1 (Fig. 1a; Supplementary material Figs. A(a) and A(b)) and SAM2 (Supplementary material Figs. $A(c)$ and $A(d))$, indicated that $0.65 \mathrm{nM}$ was the lowest concentration that could be visually differentiated, and $2.6 \mathrm{nM}$ for SAM-3 (Fig. 1b; Supplementary material Figs. $A(e)$ and $A(f))$. The curves corresponding to the lowest distinguishable concentration, representing the detection limit, have been marked with an asterisk.

Glycopeptide 1 was also immobilized on SAM-4 (Table 1), containing a 9:1 molar ratio of 3-MPOH/11-MUA. Binding data were collected at different concentrations $(4.2-0.008 \mathrm{nM})$, following the same protocol as described for SAM-1-3. As can be seen from Fig. 1c (Supplementary material Figs. $\mathrm{A}(\mathrm{g})$ and $\mathrm{A}(\mathrm{h})$ ), $0.13 \mathrm{nM}$ was the low-
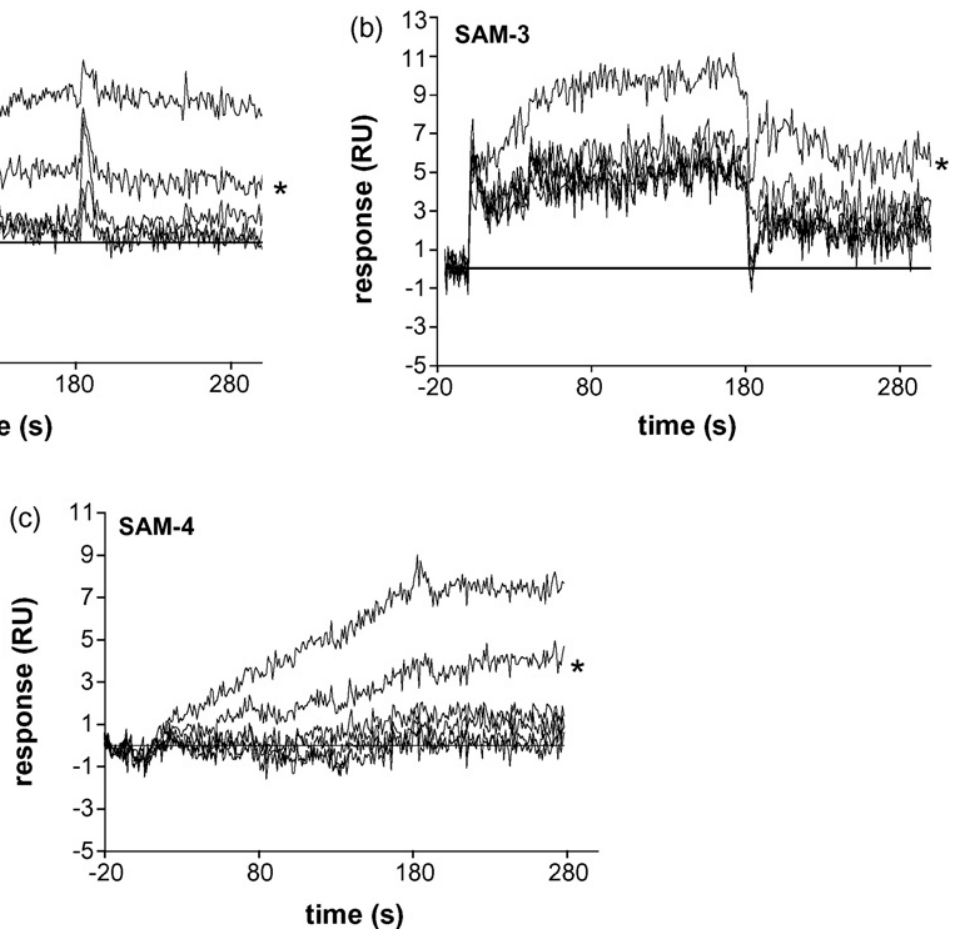

Fig. 1. (a) Glycopeptide 1, immobilized on SAM-1 (700 RU). Concentration-dependent overlay plots were made of soluble RCA 120 binding at 10 different concentrations from 41.7 to $0.08 \mathrm{nM}$ (2-fold dilution), of which the five lowest concentrations (1.3-0.08 nM) are depiccted. (b) Glycopeptide 1, immobilized on SAM-3 (300 RU). The analyzed concentration range is as in (a), of which the six lowest concentrations (2.6-0.08 nM) are depicted. (c) Glycopeptide 1, immobilized on SAM-4 (930 RU). Ten different $\mathrm{RCA}_{120}$ concentrations were analyzed from 4.2 to $0.008 \mathrm{nM}$, of which the six lowest concentrations $(0.26-0.008 \mathrm{nM})$ are depicted. The curves corresponding to the lowest distinguishable concentration, representing the detection limit, are marked with an asterisk. 
est distinguishable concentration, indicating that this SAM is the most sensitive biosensor for the detection of $\mathrm{RCA}_{120}$.

The efficiency of ligand immobilization on the acid thiols, and thereby the sensitivity of detection, is affected by a number of factors. First, hydroxyl-terminated SAMs have a lower level of non-specific analyte binding than carboxyl-terminated SAMs (Silin et al., 1997). Second, the steric hindrance (Patel et al., 1998; Lee et al., 2005) and electrostatic repulsion of the acid thiols in mixed SAMs are lower than in SAMs consisting of acid thiols only. This improves the accessibility of the carboxyl-terminated alkanethiols for the activation and subsequent ligand coupling. Third, a mixture of short hydroxyl-terminated alkanethiols and long carboxyl-terminated alkanethiols also enhances the accessibility of the acid thiols. Therefore, the optimum accessibility, and sensitivity, is achieved by fine-tuning the length and ratio of hydroxyland carboxyl-terminated alkanethiols, rather than by increasing the absolute number of acid thiols that are present on the SAM. In our study, comparison of the SAMs containing an 8:2, 9:1, and 95:5 11-MUOH/11-MUA molar ratio shows that a good sensitivity is obtained using SAMs with a 9:1 and 8:2 11-MUOH/11-MUA ratio (Table 1). Reducing the spacer length, i.e. using 3-MPOH instead of 11-MUOH, further increased the accessibility of the carboxylic acids for ligand immobilization and analyte binding. This resulted in a 5-fold improvement of the sensitivity for the detection of $\mathrm{RCA}_{120}$ (i.e. $0.65 \mathrm{nM}$ on SAM-2 versus $0.13 \mathrm{nM}$ on SAM-4). Interestingly, comparison of the shape of the various curves (for detailed pictures, see Supplementary material Fig. A) reveals that the kinetics of the binding of $\mathrm{RCA}_{120}$ to immobilized $\mathbf{1}$ is affected by the 11-MUOH/11-MUA ratio and the length of the hydroxylterminated alkanethiol (11-MUOH versus 3-MPOH). Previously, monovalent binding of (Gal-)CYPY(Gal-)C-GPPFPFK to immobilized $\mathrm{RCA}_{120}$ resulted in a $K_{\mathrm{A}}$ of $3.81 \pm 0.04 \times 10^{4} \mathrm{M}^{-1}$ (Maljaars et al., 2006). Qualitative assessment of the binding kinetics reported here suggests that the interaction of soluble RCA $\mathrm{A}_{120}$ with immobilized 1 is much stronger. Since $\mathrm{RCA}_{120}$ is a bivalent protein, the distance between the individual peptides on SAM-1 and SAM-2 probably allows bivalent binding, whereas bivalent binding is hampered on SAM-3, which has a lower peptide density, resulting in faster kinetics. SAM-4 may have both an optimum peptide density for bivalent binding of $\mathrm{RCA}_{120}$, and an improved accessibility of $\mathbf{1}$, which allows a more efficient analyte binding with a very slow association and dissociation rate, thereby enhancing the sensitivity of this biosensor. Finally, it should be noted that plots of $\mathrm{dRU} / \mathrm{d} t$ versus the $\mathrm{RCA}_{120}$ concentration are linear and slopes increase upon an enhanced sensitivity (Supplementary material Fig. $A(i))$.

\subsection{Detection of $h-G a l-1$ on SAM-5}

Galectins have attracted increasing medical attention, because these endogenous lectins with affinity for $\beta$-galactosides are involved in tumor progression and inflammatory diseases (Villalobo et al., 2006; André et al., 2007b). Consequently, their detection in extract or serum samples may reveal pertinent clinical information, warranting respective development of biosensor techniques. Previously, it has been reported that galectin concentrations in sera of cancer patients and healthy controls vary between $74 \mathrm{pM}$ (Saussez et al., unpublished results) and $32 \mathrm{nM}$ (Iurisci et al., 2000).

To assess the performance of the SAM-based biosensors used for $\mathrm{RCA}_{120}$ in the detection of a human lectin, the sensitivity of this type of biosensor was evaluated with the galectin h-Gal-1 as model. This lectin belongs to the prototype group, and forms a homodimer in solution via hydrophobic interactions and hydrogen bonds (López-Lucendo et al., 2004). Glycopeptide 2 was immobilized on

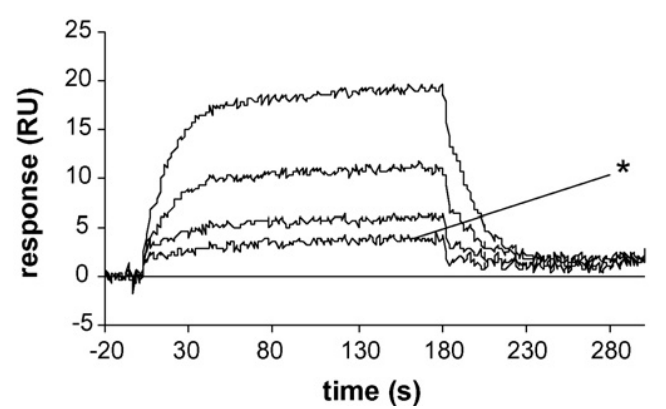

Fig. 2. Glycopeptide 2, immobilized on SAM-5 (1256 RU). Concentration-dependent overlay plots were made of soluble h-Gal-1 binding at ten different concentrations from 500 to $0.9 \mathrm{nM}$ (2-fold dilution), of which the four lowest h-Gal-1 concentrations (7.8-0.9 nM) are depicted. The curve corresponding to the lowest distinguishable concentration, representing the detection limit, is marked with an asterisk.

SAMs containing mixtures of hydroxyl- and carboxyl-terminated alkanethiols, analogous to SAM-1-4. The significant non-specific binding of h-Gal-1 that was initially observed on the underivatized SAM of the reference surface could be completely eliminated by immobilizing ethanolamine. The correlation between the sensitivity of the biosensor and the composition of the SAM (i.e. the length and ratio of hydroxyl- and carboxyl-terminated alkanethiols) was similar for $\mathrm{RCA}_{120}$ and h-Gal-1 (data not shown). The most sensitive biosensor was obtained with a SAM consisting of a 9:1 ratio of 3-MPOH/11-MUA (SAM-5; Table 1). Binding data were obtained at different h-Gal-1 concentrations (500-0.9 nM) at a flow rate of $5 \mu \mathrm{min}^{-1}$. Visual inspection of the curve in Fig. 2 (see also Supplementary material Figs. $\mathrm{B}(\mathrm{a})$ and $\mathrm{B}(\mathrm{b})$ ) indicated that the lowest distinguishable h-Gal-1-concentration of the series tested (marked with an asterisk) surpassed the $1 \mathrm{nM}$ threshold with $0.9 \mathrm{nM}$ on this SAM. It can be expected that the actual detection limit might even be slightly lower. For the h-Gal-1 binding to immobilized 2 , a $K_{\mathrm{D}}$ of $0.36 \pm 0.04 \mu \mathrm{M}$ was obtained from a non-linear fit of the data in a plot of $R_{\mathrm{eq}}$ versus the h-Gal-1 concentration (Supplementary material Fig. $\mathrm{B}(\mathrm{c})$ ). In contrast, an $\mathrm{IC}_{50}$ value of $235 \pm 15 \mu \mathrm{M}$ was obtained for (Lac-)TMRA(Lac-)TCha-GPPFPFK from competition experiments by allowing soluble glycopeptide to compete with immobilized asialofetuin in the presence of soluble h-Gal-1 (Maljaars et al., unpublished results). This indicates that the multivalent presentation of $\mathbf{2}$ on SAM-5 allows a stronger interaction with h-Gal-1 when compared to the glycopeptide h-Gal-1 interaction in solution. A possible explanation could be bivalent binding of h-Gal-1 to SAM-5, however, slower kinetics than observed in Fig. 2 would then be more likely.

As can be seen from Table 1, the best biosensor for the detection of $\mathrm{RCA}_{120}$ was more sensitive for $\mathrm{RCA}_{120}$ (detection limit $0.13 \mathrm{nM}$ ) than a similar biosensor for the detection of h-Gal-1 (detection limit $0.9 \mathrm{nM}$ ). This can be attributed, at least in part, to the difference in molecular mass $\left(120 \mathrm{kDa}\right.$ for $\mathrm{RCA}_{120}$ versus $30 \mathrm{kDa}$ for h-Gal-1), since SPR-detection is based on mass-dependent changes in refractive index at the sensor chip surface.

\subsection{Detection of carbohydrate-binding proteins in protein mixtures}

To assess the performance of the best biosensors described above in detecting low concentrations of analyte in the presence of other proteins, a model protein mixture containing ovalbumin, BSA, RNase $B$, and trypsin inhibitor (1:1:1:1 molar ratio) spiked with $\mathrm{RCA}_{120}$ or h-Gal-1 was tested.

In the case of $\mathrm{RCA}_{120}$, stock solutions of $4.2 \mathrm{nM} \mathrm{RCA} \mathrm{A}_{120}$ were prepared containing a 10-, 100-, 1000-, and 10,000-fold excess, respectively, of each component of the protein mixture. Dilution 


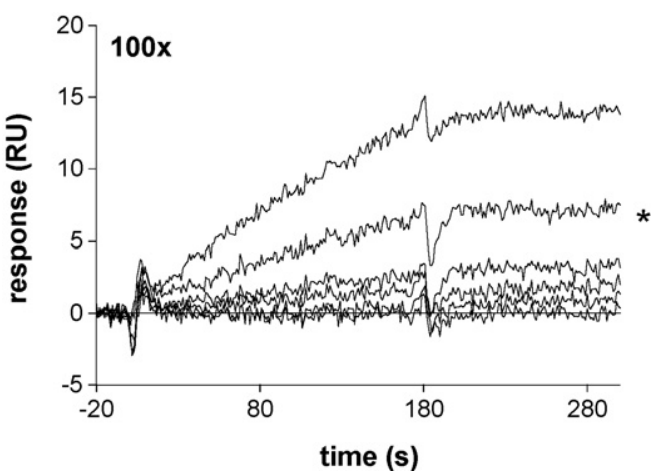

Fig. 3. Glycopeptide 1, immobilized on SAM-6 (1032 RU). Concentration-dependent overlay plots were made of soluble $\mathrm{RCA}_{120}$ binding at 10 different concentrations from 4.2 to $0.008 \mathrm{nM}$ (2-fold dilution), containing a 100 -fold molar excess of each component of the protein mixture (1:1:1:1 BSA/ovalbumin/RNase B/trypsin inhibitor). The curves resulting from the six lowest concentrations $(0.26-0.008 \mathrm{nM})$ are depicted. The curve corresponding to the lowest distinguishable concentration, representing the detection limit, is marked with an asterisk.

series were prepared (4.2-0.008 $\left.\mathrm{nM} \mathrm{RCA}_{120}\right)$ from these stock solutions, and SPR measurements were carried out at a flow rate of $5 \mu \mathrm{min}^{-1}$ on glycopeptide 1-derivatized SAM-6 (comparable to SAM-4; Table 1). As can be seen from Fig. 3 (for full details, see Supplementary material Figs. $C(a-d)$ ), the detection limit of $0.13 \mathrm{nM}$ (marked with an asterisk) was not influenced by protein concentrations up to 100 -fold higher than the $\mathrm{RCA}_{120}$ concentration. Additional experiments with a 1000 -fold (Supplementary material Figs. C(e) and C(f)) or a 10,000-fold (data not shown) excess of each component of the protein mixture showed a significant disturbance of the association phase due to a major contribution of the protein mixture to the bulk effect.

For h-Gal-1, stock solutions of $500 \mathrm{nM}$ h-Gal-1 were prepared containing an equimolar amount, as well as a 10-, 50-, and 100-fold excess of each component of the protein mixture. Dilution series (500-0.9 nM h-Gal-1) were prepared from these stock solutions, and were measured at a flow rate of $5 \mu \mathrm{min}^{-1}$ on glycopeptide 2derivatized SAM-5. It turned out that an equimolar amount of each component of the protein mixture relative to h-Gal-1 did not influence the signal (for full details, compare Supplementary material Figs. B and D). However, the shift in response at the end of the association phase indicated that the bulk effect already interfered with the h-Gal-1 response at a 10-fold excess (Supplementary material Figs. $D(c)$ and $D(d)$ ), but, without affecting the detection limit of $0.9 \mathrm{nM}$. At a $50-$ and 100 -fold excess of each component of the protein mixture, the huge contribution of the protein mixture to the bulk effect (Supplementary material Figs. $\mathrm{D}(\mathrm{e}-\mathrm{h})$ ) precluded the determination of the detection limit. In the latter experiments, the absolute amount of protein corresponds to that of the 1000 to 10,000 -fold excess relative to $\mathrm{RCA}_{120}$, where the same effect was observed. Again, the size of the analyte may be of influence, since $\mathrm{RCA}_{120}$ has a higher molecular mass than all proteins used in the protein mixture, whereas the molecular masses of h-Gal1 and the components of the protein mixture are in the same range.

Comparison of the observed detection limit, even in the presence of 10-fold excess of contaminating proteins, with the above-mentioned biologically relevant galectin levels indicates that the sensitivity would be sufficient for detecting the concentration at which galectins function in cells or tissues, as well as elevated galectin levels in patient sera. However, in view of possibly higher amounts of contaminating proteins that could influence the detection limit, biological sample pre-treatment (partial purification/concentration) needs further attention.

\section{Concluding remarks}

This work compares several SPR-based biosensors containing mixed alkanethiol SAMs with respect to their performance in detecting two different CBPs in solution on a Biacore 2000 instrument. The results clearly demonstrate the potential of these types of biosensors for the sensitive detection of CBPs in solution. Excellent sensitivities were obtained using glycopeptide-derivatized SAMs with a 9:1 3-MPOH/11-MUA ratio, resulting in detection limits of 0.13 and at least $0.9 \mathrm{nM}$ for the detection of $\mathrm{RCA}_{120}$ and h-Gal-1, respectively.

The experiments with $\mathrm{RCA}_{120}$ and $\mathrm{h}-\mathrm{Gal}-1$ containing an excess of a protein mixture indicated that the presence of interfering compounds at a certain concentration can affect the sensitivity of the biosensor. Nevertheless, this method may be applied for the quantification of CBPs in solution by transformation of a dilution series into a calibration curve. The biosensors may, for example, be clinically applicable for the quantification or monitoring of lectin levels in patient samples or in biological materials, when excluding a biohazard is an issue. Moreover, the coupling of this method to, for example, mass spectrometry may increase the applicability and versatility, which would allow the determination of the presence of multiple analytes with the same specificity in a mixture of proteins. Finally, another set-up, in which glycopeptides with a different carbohydrate are immobilized in different flow cells, may allow the simultaneous detection of analytes that possess a different carbohydrate specificity.

\section{Acknowledgement}

We thank Dstl Porton Down, UK, for providing the financial support for this study, also supported by the research initiative LMUexcellent.

\section{Appendix A. Supplementary data}

Supplementary data associated with this article can be found, in the online version, at doi:10.1016/j.bios.2008.03.014.

\section{References}

André, S., Kaltner, H., Furuike, T., Nishimura, S.-I., Gabius, H.-J., 2004. Bioconjugate Chem. 15 (1), 87-98.

André, S., Maljaars, C.E.P., Halkes, K.M., Gabius, H.-J., Kamerling, J.P., 2007a. Bioorg. Med. Chem. Lett. 17 (3), 793-798.

André, S., Sanchez-Ruderisch, H., Nakagawa, H., Buchholz, M., Kopitz, J., Forberich, P., Kemmner, W., Böck, C., Deguchi, K., Detjen, K.M., Wiedenmann, B., von Knebel Doeberitz, M., Gress, T.M., Nishimura, S., Rosewicz, S., Gabius, H.-J., 2007b. FEBS J. 274 (13), 3233-3256

Blixt, O., Head, S., Mondala, T., Scanlan, C., Huflejt, M.E., Alvarez, R., Bryan, M.C., Fazio, F., Calarese, D., Stevens, J., Razi, N., Stevens, D.J., Skehel, J.J., van Die, I., Burton, D.R., Wilson, I.A., Cummings, R., Bovin, N., Wong, C.-H., Paulson, J.C., 2004. Proc. Natl. Acad. Sci. U.S.A. 101 (49), 17033-17038.

Bourne, Y., Astoul, C.H., Zamboni, V., Peumans, W.J., Menu-Bouaouiche, L., van Damme, E.J.M., Barre, A., Rouge, P., 2002. Biochem. J. 364 (1), 173-180.

Castillo, J., Gaspar, S., Leth, S., Niculescu, M., Mortari, A., Bontidean, I., Soukharev, V., Dorneanu, S.A., Ryabov, A.D., Csoregi, E., 2004. Sens. Actuators B102 (2), 179-194.

Choi, S.H., Lee, J.W., Sim, S.J., 2005. Biosens. Bioelectron. 21 (2), 378-383.

Chou, S.F., Hsu, W.L., Hwang, J.M., Chen, C.Y., 2004. Biosens. Bioelectron. 19 (9) 999-1005.

Clark Jr., L.C., Lions, C., 1962. Ann. N. Y. Acad. Sci. 102, 29-45

Cullum, B.M., Vo-Dinh, T., 2000. Trends Biotechnol. 18 (9), 388-393.

Dillon, P.P., Killard, A.J., Daly, S.J., Leonard, P., O'Kennedy, R., 2005. J. Immunol. Methods $296(1-2), 77-82$.

Dyukova, V.I., Shilova, N.V., Galanina, O.E., Rubina, A.Y., Bovin, N.V., 2006. Biochim. Biophys. Acta 1760 (4), 603-609.

Feizi, T., Fazio, F., Chai, W.C., Wong, C.-H., 2003. Curr. Opin. Struct. Biol. 13 (5), 637-645.

Gustavsson, E., Bjurling, P., Sternesjo, A., 2002. Anal. Chim. Acta 468 (1), 153-159.

Haasnoot, W., Loomans, E.E.M.G., Cazemier, G., Dietrich, R., Verheijen, R., Bergwerff, A.A., Stephany, R.W., 2002. Food Agric. Immunol. 14 (1), 15-27. 
Halkes, K.M., St. Hilaire, P.M., Crocker, P.R., Meldal, M., 2003. J. Comb. Chem. 5 (1), $18-27$.

Hsieh, H.V., Pfeiffer, Z.A., Amiss, T.J., Sherman, D.B., Pitner, J.B., 2004. Biosens. Bioelectron. 19 (7), 653-660.

Indyk, H.E., Filonzi, E.L., 2005. Int. Dairy J. 15 (5), 429-438.

Ista, L.K., Mendez, S., Perze-Luna, V.H., Lopez, G.P., 2001. Langmuir 17 (9), 2552-2555. Iurisci, I., Tinari, N., Natoli, C., Angelucci, D., Cianchetti, E., Iacobelli, S., 2000. Clin. Cancer Res. 6 (4), 1389-1393.

Jiang, T.S., Minunni, M., Wilson, P., Zhang, J., Turner, A.P.F., Mascini, M., 2005. Biosens. Bioelectron. 20 (10), 1939-1945.

Lee, J.W., Sim, S.J., Cho, S.M., Lee, J., 2005. Biosens. Bioelectron. 20 (7), 1422-1427.

Liu, X., Sun, Y., Song, D.Q., Zhang, Q.L., Yuan, T., Bi, S.Y., Zhang, H.Q., 2004. Anal. Biochem. 333 (1), 99-104

López-Lucendo, M.F., Solís, D., André, S., Hirabayashi, J., Kasai, K., Kaltner, H., Gabius, H.-J., Romero, A., 2004. J. Mol. Biol. 343 (4), 957-970.

Love, K.R., Seeberger, P.H., 2002. Angew. Chem. Int. Ed. 41 (19), 3583-3586.

Lu, J.Z., Rosenzweig, Z., 2000. Fresenius J. Anal. Chem. 366 (6-7), 569-575.

Maljaars, C.E.P., de Oude, W.L., Halkes, K.M., Haseley, S.R., Upton, P.J., McDonnell, M.B., Kamerling, J.P., 2006. J. Comb. Chem. 8 (6), 812-819.

Miura, Y., Kimura, S., Imanishi, Y., Umemura, J., 1999. Langmuir 15 (4), 1155-1160.
Muller-Renaud, S., Dupont, D., Dulieu, P., 2005. J. Dairy Res. 72 (1), 57-64.

Oh, B.K., Kim, Y.K., Park, K.W., Lee, W.H., Choi, J.W., 2004. Biosens. Bioelectron. 19 (11), 1497-1504

Patel, N., Davies, M.C., Heaton, R.J., Roberts, C.J., Tendler, S.J.B., Williams, P.M., 1998. Appl. Phys. A: Mater. Sci. Process. 66 (Suppl. 1), S569-S574.

Shinohara, Y., Kim, F., Shimizu, M., Goto, M., Tosu, M., Hasegawa, Y., 1994. Eur. J. Biochem. 223 (1), 189-194.

Silin, V., Weetall, H., Vanderah, D.J., 1997. J. Colloid Interface Sci. 185 (1), 94-103.

Soh, N., Tokuda, T., Watanabe, T., Mishima, K., Imato, T., Masadome, T., Asano, Y., Okutani, S., Niwa, O., Brown, S., 2003. Talanta 60 (4), 733-745.

St. Hilaire, P.M., Lowary, T.L., Meldal, M., Bock, K., 1998. J. Am. Chem. Soc. 120 (51), 13312-13320.

Tamada, K., Ishida, T., Knoll, W., Fukushima, H., Colorado, R., Graupe, M., Shmakova, O.E., Lee, T.R., 2001. Langmuir 17 (6), 1913-1921.

Villalobo, A., Nogales-Gonzáles, A., Gabius, H.-J., 2006. Trends Glycosci. Glycotechnol. 18 (99), 1-37.

Vo-Dinh, T., Cullum, B., 2000. Fresenius J. Anal. Chem. 366 (6-7), 540-551.

Zhang, Y., Luo, S., Tang, Y., Yu, L., Hou, K.-Y., Cheng, J.-P., Zeng, X., Wang, P.G., 2006. Anal. Chem. 78 (6), 2001-2008. 